\title{
Physicochemical and Microstructural Properties of Refined Palm Oil-Palm Kernel Oil Blends Following Chemical Interesterification
}

\author{
Siti Hazirah Mohamad Fauzi, Norizzah Abd. Rashid, and Zaliha Omar
}

\begin{abstract}
This study evaluated the effects of chemical interesterification (CIE) using sodium methoxide on refined palm oil (PO), palm kernel oil (PKO) and their constituent blends formulated using a mixture design based on simplex-lattice (Design Expert 8.0.4 Stat-Ease Inc., Minneapolis, 2010). The slip melting point (SMP), solid fat content (SFC), triacylglycerol composition (TAG), and microstructural of non-interesterified and interesterified blends were determined. Results indicated that all the interesterified PO and blends had higher SMP and SFC than non-interesterified blends except for PKO. Chemical interesterification also altered the distributions of fatty acids in TAG of blends and consequently modifies the microstructure of PO, PKO and blends following interesterification. This study has demonstrated chemical interesterification is effective in modifying the physicochemical properties of palm oil and palm kernel oil blends.
\end{abstract}

Index Terms-Chemical interesterification, design-expert, palm oil, palm kernel oil.

\section{INTRODUCTION}

Palm oil contains a mixture of high and low melting triacylglycerols. At ambient temperatures, higher melting triacylglycerols will crystallize into a solid fraction called stearin, while the lower melting triacylglycerols will remain in a liquid form called olein [1]. Palm kernel oil (PKO) contains short and medium chain length fatty acids similar to those present in butterfat, and therefore helps to give the right mouth feel and melting characteristics, and ensures the stable beta prime $\left(\beta^{\prime}\right)$ crystalline form necessary for a fine structure of products [2].

Most native vegetable oils have limited applications in their original forms due to their specific chemical composition. To widen their commercial use, vegetable oils are modified either physically (by fractionation/blending) or chemically by hydrogenation/interesterification [3]-[5].

Chemical interesterification (CIE) has received much interest in the edible oil industry and has long been used to modify oils and fats into functional products. It modifies the physical properties of oils by rearranging the distribution of fatty acids on the glycerol backbone without changing their chemical composition [6]. The recent interest in minimizing

Manuscript received April 20, 2015; revised June 27, 2015.

Siti Hazirah Mohamad Fauzi, and Zaliha Omar are with the Malaysian Palm Oil Board, Malaysia (e-mail: sitihazirah@mpob.gov.my, zaliha@mpob.gov.my).

Norizzah Abd. Rashid is with the Faculty of Applied Sciences, Universiti Teknologi MARA, Malaysia (e-mail: norizzah850@ salam.uitm.edu.my). the trans fatty acid content of fat products especially in the production of margarine has created attention on interesterification and palm oil products as a source of trans-free solid fat in replacing hydrogenated fats [2].

Hence, in this study, blends of refined, bleached and deodorized palm oil (PO) and palm kernel oil (PKO) were blended and chemically interesterified. The objectives were to determine the physical and chemical properties of $\mathrm{PO}$ and PKO by blending at certain ratios according to the Design Expert version 8.0.4 (2010), and to compare the physicochemical properties of the blends before and after chemical interesterification.

\section{MATERIALS AND METHOD}

\section{A. Materials}

Refined, bleached, and deodorized (RBD) PO and PKO were obtained from Golden Jomalina Sdn Bhd, Klang, Malaysia. The oils and fats were stored at $0{ }^{\circ} \mathrm{C}$ prior to use. All the chemicals used were either analytical or high-performance liquid chromatography grade.

\section{B. Blend Preparation}

The PO and PKO were melted at $60{ }^{\circ} \mathrm{C}$ in an oven prior to use. Based on the Simplex-Lattice Mixture Design (Design Expert 8.0.4, 2010), three binary blends were prepared in the following mass $(\mathrm{w} / \mathrm{w})$ ratios of PO to PKO (PO:PKO) with $25 \%$ of increments; 25:75, 50:50, and 75:25.

\section{Chemical Interesterification (CIE)}

The chemical interesterification process was carried out according to [7]. The fat blend was dried under nitrogen at $100{ }^{\circ} \mathrm{C}$ for $60 \mathrm{~min}$. After lowering the temperature to $80^{\circ} \mathrm{C}$, $0.2 \%$ of sodium methoxide powder (catalyst) was added. The mixture was heated to $110^{\circ} \mathrm{C}$ under nitrogen for $60 \mathrm{~min}$ with vigorous stirring. It was then cooled to $70{ }^{\circ} \mathrm{C}$ and $5 \%$ citric acid solution was added to inactivate the catalyst. After the mixture was stirred mechanically at $70{ }^{\circ} \mathrm{C}$ for $15 \mathrm{~min}$, the excess citric acid and sodium methoxide were removed with warm water washes. Finally the interesterified oil was filtered through a Whatman \#4 filter paper. The non-interesterified oil is abbreviated as NIE and interesterified oil as CIE.

\section{Triacylglycerol Composition (TAG)}

The TAG composition of the blends were analysed in a reversed phase high-performance liquid chromatograph (Gilson, Villiersel-Bel, France). A Lichrosphere RP-18 
column $(250 \mathrm{~mm} \times 4 \mathrm{~mm})$ of $5-\mu \mathrm{m}$ particle size (Merck, Darmstadt, Germany) with acetone/acetonitrile (75:25\% v/v) as the eluent at a flow rate of $1.0 \mathrm{ml} / \mathrm{min}$ and a refractive index detector was used.

\section{E. Slip Melting Point (SMP)}

The SMP of the NIE and CIE blends was determined according to [7]. Three replicates of this analysis were performed.

\section{F. Solid Fat Content (SFC)}

The SFC of the blends was measured using a Bruker Minispec pulsed Nuclear Magnetic Resonance (pNMR) spectrometer (Karlsruhe, Germany). Measurements were carried out at $5-50{ }^{\circ} \mathrm{C}$ with $5{ }^{\circ} \mathrm{C}$ interval [7].

\section{G. Microstructure}

The microstructures of blends were determined using Polarized Light Microscope (Wetzlar, Germany). A small droplet (about $10 \mu \mathrm{l}$ ) of melted fat was placed on a glass slide and covered with a glass slip. The sample was first heated to $70{ }^{\circ} \mathrm{C}$ for $10 \mathrm{~min}$ and then rapidly cooled $\left(50^{\circ} \mathrm{C} / \mathrm{min}\right)$ to $20^{\circ} \mathrm{C}$ The sample was tempered at $20{ }^{\circ} \mathrm{C}$ for $10 \mathrm{~min}$ prior to measurement. A Leica DMLP polarized light microscope equipped with a Linkam THMS 600 temperature controller stage and a JVC 3-CCD colour video camera was used. Temperature was thermostatically controlled by a Linkam TP 94 multiramp temperature programmer and LNP automatic cooling system (Linkam, Tadworth, Surrey, United Kingdom) Liquid nitrogen was used as the coolant. The photomicrograph of the crystal was taken at $200 \mathrm{x}$ magnification.

\section{RESULTS AND DISCUSSION}

\section{A. Triacylgylcerol Composition (TAG)}

Table I shows the TAG composition of PO and PKO as well as their constituent blends before and after chemical interesterification. The main TAG of palm oil (PO) were POP, POO, PLO, PLP and PPP where $\mathrm{P}$ is palmitic acid, $\mathrm{L}$ is linoleic acid and $\mathrm{O}$ is oleic acid while palm kernel oil (PKO) contained a wide range of medium- chain TAG species such as LaLaLa, LaLaM, LaLaP, CaLaLa, CLaLa and LaLaO, where $\mathrm{La}$ is lauric acid, $\mathrm{M}$ is myristic acid, $\mathrm{Ca}$ is caprylic acid, $\mathrm{C}$ is capric acid and $\mathrm{O}$ is oleic acid. The original $\mathrm{PO}$ and $\mathrm{PKO}$ generated only few changes in TAG after CIE as compared to their blends. This apparently because of intraesterification did not result in much change in the TAG composition.

The changes in TAG proportions due to IE reaction were more pronounced in the binary blends due to the presence of varied TAG species in the mixtures. Randomisation led to the formation of a new TAG that could not be identified due to the complex interaction of the mixture blends. However, the TAG profile of the IE blends showed an even TAG distribution than the starting blends, as the relative concentration of several TAG increased; while others decreased as shown in Table I. This result is consistent with findings in [8].

\section{B. Slip Melting Point (SMP)}

As observed in Fig. 1 the chemically interesterified blends (CIE) had higher SMP than non-interesterified blends at all ratios examined except for $100 \%$ of PKO. The palm oil containing approximately same amounts of saturated and unsaturated fatty acid within the TAG molecule.

TABLE I: TRIACYLGLYCEROL COMPOSITION OF PO, PKO AND BINARY BLENDS BEFORE AND AFTER CIE

\begin{tabular}{|c|c|c|c|c|}
\hline \multirow[b]{2}{*}{ TAG } & \multicolumn{2}{|c|}{ PO } & \multicolumn{2}{|c|}{ PKO } \\
\hline & NIE & CIE & NIE & CIE \\
\hline CLaLa & ND & ND & 6.01 & 5.01 \\
\hline CaLaLa & ND & ND & 8.49 & 6.30 \\
\hline LaLaLa & ND & ND & 15.79 & 13.57 \\
\hline LaLaM & ND & ND & 15.79 & 13.57 \\
\hline $\mathrm{LaLaO}$ & ND & ND & 4.94 & 12.64 \\
\hline $\mathrm{LaLaP}$ & ND & ND & 8.85 & 10.10 \\
\hline $\mathrm{LaOM}$ & ND & ND & 4.61 & 7.70 \\
\hline $\mathrm{LaPM}$ & ND & ND & 4.36 & 5.33 \\
\hline $\mathrm{LaOO}$ & ND & ND & 3.70 & 2.85 \\
\hline $\mathrm{LaOP}$ & ND & ND & 4.25 & 4.10 \\
\hline LaPP & ND & ND & 2.39 & 2.49 \\
\hline OLO & 1.80 & 4.53 & 0.99 & 0.87 \\
\hline PLO & 9.87 & 10.55 & 1.85 & 1.59 \\
\hline OOO & 4.33 & 6.64 & 1.87 & 1.12 \\
\hline POO & 23.18 & 21.66 & 1.54 & 0.31 \\
\hline POP & 30.55 & 25.19 & 0.92 & 0.46 \\
\hline PPP & 5.86 & 9.46 & 1.17 & 0.47 \\
\hline SOO & 2.81 & 1.97 & 0.38 & ND \\
\hline POS & 5.64 & 4.65 & 0.37 & ND \\
\hline OLL & 0.4 & 1.07 & ND & ND \\
\hline PLL & 1.88 & 1.49 & ND & ND \\
\hline MPL & 0.53 & 0.50 & ND & ND \\
\hline PMP & 1.57 & 1.32 & ND & ND \\
\hline PPS & 1.02 & 2.42 & ND & ND \\
\hline SOS & 0.57 & 0.50 & ND & ND \\
\hline PLP & 9.14 & 6.22 & ND & ND \\
\hline Others & 0.85 & 1.83 & 4.82 & 8.44 \\
\hline
\end{tabular}

\begin{tabular}{|c|c|c|c|c|c|c|}
\hline \multirow[b]{2}{*}{ TAG } & \multicolumn{2}{|c|}{ PO:PKO (75:25) } & \multicolumn{2}{|c|}{ PO:PKO (50:50) } & \multicolumn{2}{|c|}{ PO:PKO (25:75) } \\
\hline & NIE & CIE & NIE & CIE & NIE & CIE \\
\hline CLaLa & 1.30 & 0.22 & 2.76 & 0.83 & 4.29 & 2.60 \\
\hline CaLaLa & 2.85 & 1.87 & 4.64 & 1.78 & 6.44 & 3.44 \\
\hline LaLaLa & 5.56 & 0.95 & 10.99 & 3.47 & 16.43 & 8.77 \\
\hline LaLaM & 3.68 & 1.04 & 7.53 & 3.68 & 11.47 & 7.87 \\
\hline $\mathrm{LaLaO}$ & 1.25 & 2.83 & 2.43 & 7.03 & 3.63 & 11.64 \\
\hline LaLaP & 2.19 & 2.63 & 4.19 & 6.95 & 6.28 & 9.78 \\
\hline $\mathrm{LaOM}$ & 1.37 & 2.45 & 2.04 & 4.69 & 3.12 & 6.22 \\
\hline $\mathrm{LaPM}$ & 1.08 & 2.77 & 2.28 & 5.00 & 3.86 & 5.89 \\
\hline $\mathrm{LaOO}$ & 0.91 & 4.23 & 1.67 & 5.97 & 2.9 & 4.61 \\
\hline $\mathrm{LaOP}$ & 1.16 & 10.06 & 2.03 & 11.54 & 3.23 & 8.48 \\
\hline LaPP & 2.00 & 7.39 & 1.99 & 7.23 & 2.28 & 4.73 \\
\hline OLO & 3.56 & 2.96 & ND & 1.61 & 0.97 & 1.41 \\
\hline PLO & 7.49 & 5.02 & 5.06 & 5.34 & 2.96 & 3.96 \\
\hline OOO & 3.56 & 6.58 & 3.04 & 4.48 & 2.74 & 2.70 \\
\hline POO & 17.2 & 11.04 & 12.5 & 5.09 & 7.48 & 2.26 \\
\hline POP & 22.63 & 12.24 & 16.00 & 5.31 & 8.36 & 2.23 \\
\hline PPP & 4.29 & 4.48 & 3.26 & 2.12 & 1.68 & 0.80 \\
\hline SOO & 1.76 & 1.22 & 1.19 & 0.50 & 0.85 & ND \\
\hline POS & 3.89 & 2.79 & 2.55 & 1.08 & 1.46 & 0.39 \\
\hline OLL & ND & ND & ND & ND & 0.39 & ND \\
\hline PLL & ND & ND & ND & ND & ND & ND \\
\hline MPL & ND & ND & ND & ND & ND & ND \\
\hline PMP & 1.63 & ND & 1.73 & ND & 1.79 & ND \\
\hline PPS & 0.75 & 1.63 & 0.73 & 0.79 & 0.31 & ND \\
\hline SOS & 0.49 & ND & 0.39 & ND & ND & ND \\
\hline PLP & 7.14 & ND & 4.28 & ND & 2.16 & ND \\
\hline Others & 2.26 & 15.60 & 6.72 & 15.51 & 4.93 & 12.31 \\
\hline
\end{tabular}

Notes: C, capric acid; La, lauric acid; Ca, caprylic acid; M, myristic acid; O, oleic acid; P, palmitic acid; L, linoleic acid; S, stearic acid; ND, not detectable. 


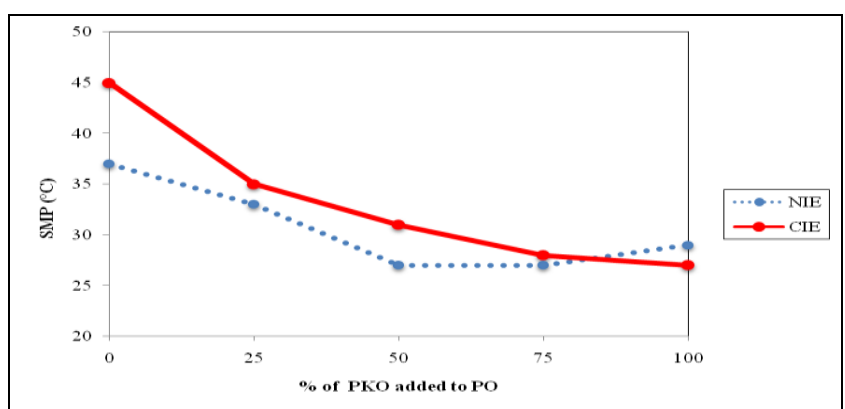

Fig. 1. The SMP of non-interesterified (NIE) and chemically interesterified (CIE) PO, PKO and their binary blends.

The high melting point of chemically-interesterified PO was due to the presence of high-melting TAGs, such as POP, POO and PLL as compared to the non-interesterified PO (Table I). As expected, the largest increase in SMP was observed in CIE PO with $45^{\circ} \mathrm{C}$. The PKO contains short- and medium-chain saturated fatty acids and the CIE PKO tended to have lower SMP than the original PKO relatively due to the randomisation and redistribution of major TAG of PKO such as LaLaLa, LaLaO, LaLaM and CLaLa after interesterification hence resulting lower slip melting point.

The increase in SMP of the interesterified blends indicated that PO blended with PKO (a liquid oil of shorter saturated fatty acids) resulted in blends with higher slip melting points than the non-interesterified blends. The SMP increased with an increase in the amount of PO in the blends due to formation of the high amount of high-melting TAGs (Table I). This observation supported by other findings [9] and [10] who reported that chemical interesterification produced a significant increase in the melting point after the reaction. The precise effect of interesterification can decrease, increase, or even present no effect on melting point are very much depends on the initial raw materials [11].

\section{Solid Fat Content (SFC)}

The SFC was dependent on both temperature and the proportion of each fat in the blend. As shown in Fig. 2b, the CIE increased the SFC at all analyzed temperatures, except for PKO. This finding agreed with the SMP results obtained. The NIE and CIE of PO-PKO blends had similar SFC profiles and their SFC increased with increasing amounts of $\mathrm{PO}$ in the blends.

The NIE PKO (Fig. 2a) had the highest SFC (77.81\%) at the starting temperature and followed by CIE PKO with $75.76 \%$ (Fig. 2b) but both completely liquefied at $30{ }^{\circ} \mathrm{C}$. This can be explained due to the chemical composition of PKO which contains wide range of medium-chain TAGs (Table I).

Before chemical interesterification, the PO and binary blends contained noticable proportions of higher melting triacylglycerols, such as POO, PPO, and PPL as tabulated in Table I. After the reaction, randomization and redistribution of high-melting TAG of PO with medium-melting TAG of $\mathrm{PKO}$ resulted in higher SFC curves of chemically-interesterified PO and blends compared to the original.

Fig. 2(c) shows there was a slight eutectic interaction in the NIE PO and PKO blends at 10 to $20^{\circ} \mathrm{C}$. Eutectic behaviour occurs in the non-interesterified blends due to the differences in the molecular size of the TAGs, shape and polymorph of the crystals between the two types of fat. This shows that the $\mathrm{PO}$ and PKO are incompatible with each other. After the CIE reaction, eutectic interaction was only observed at $10{ }^{\circ} \mathrm{C}$, as shown in Fig. 2(d). At this temperature, the reduced eutectic behavior is due to the presence of residual amounts of TAG with long and medium chain fatty acids. This result was consistent with [6] and [12] findings, whereby interesterification would eliminate or reduce eutectic interactions in a eutectic mixture. The eutectic effect is desirable if this blend is going to be used for the production of margarine and shortening [12].
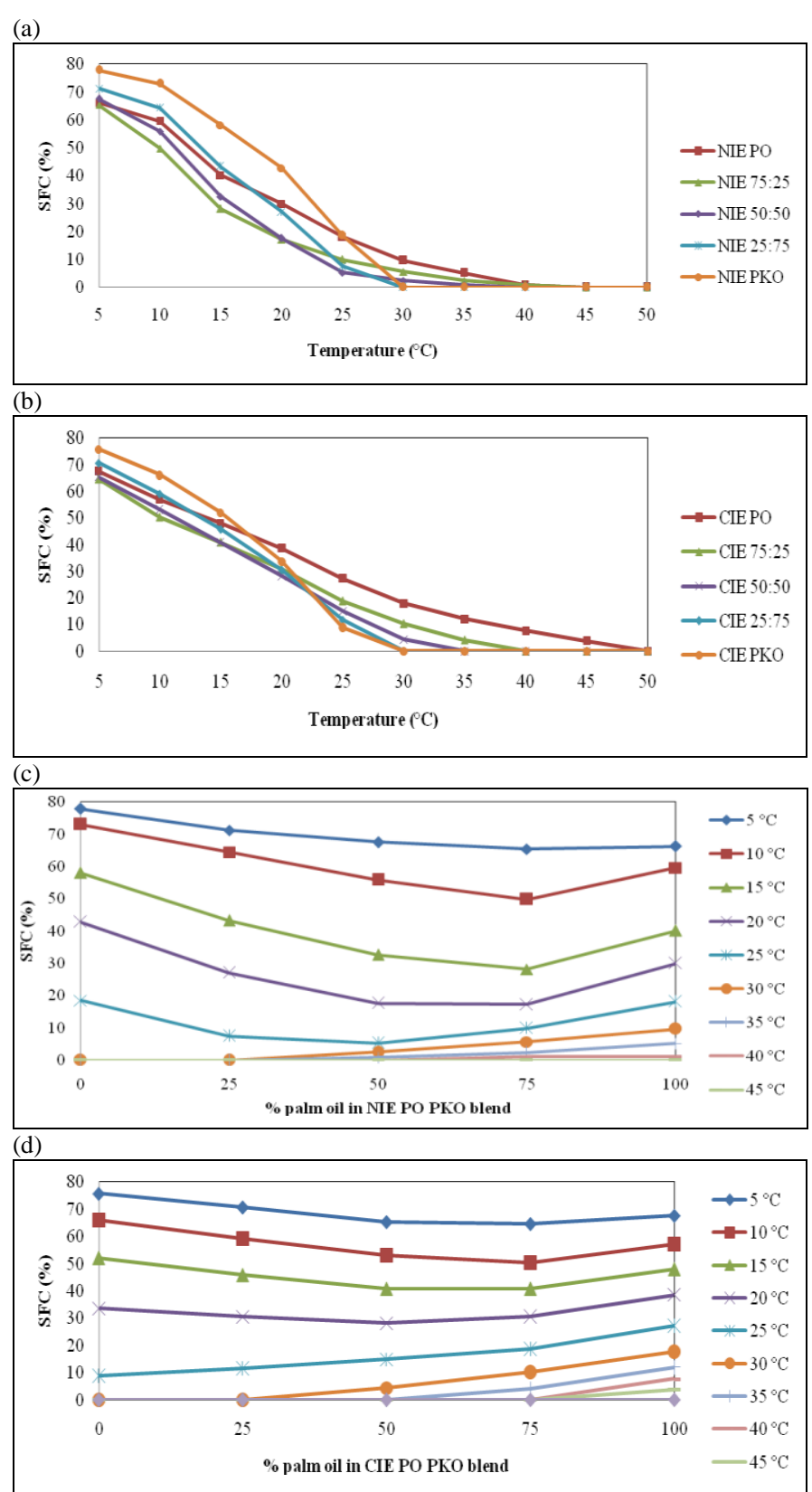

Fig. 2. (a) The solid fat contents (SFC) of non-interesterified (NIE) PO, PKO and binary blends. (b) The SFC of chemically-interesterified (CIE) PO, PKO and binary blends. (c) Eutectic effects of NIE PO, PKO and binary blends at different temperatures. (d) Eutectic effects of CIE PO, PKO and binary blends at different temperatures.

\section{Microstructure}

Fig. 3 shows the crystal morphology of PO, PKO and binary blends before (NIE) and after chemical interesterification (CIE) at $20^{\circ} \mathrm{C}$. As observed, after the IE, it 
promotes an alteration in the microstructure. The NIE PO (coded a) consists of small plate-like crystals. After CIE (coded b) an increase in number crystal-network distribution. The NIE PKO (coded i) consists large and loosely spherulitic crystal but after CIE (coded j) smaller and more crystals were formed.

Addition of $25 \%$ PKO into the blends resulted in the mixtures of plate-like and spherulitic shaped crystals (coded c). As the concentration of PKO in the blend increased, larger spherulitic crystals were observed. In general, interesterification produced blends with increasing number of crystals with different sizes. Crystal size properties are essential for final product consistency and acceptability as smaller crystals lead to firmer fat products, whereas larger crystals will produce a sandy mouthfeel [13].

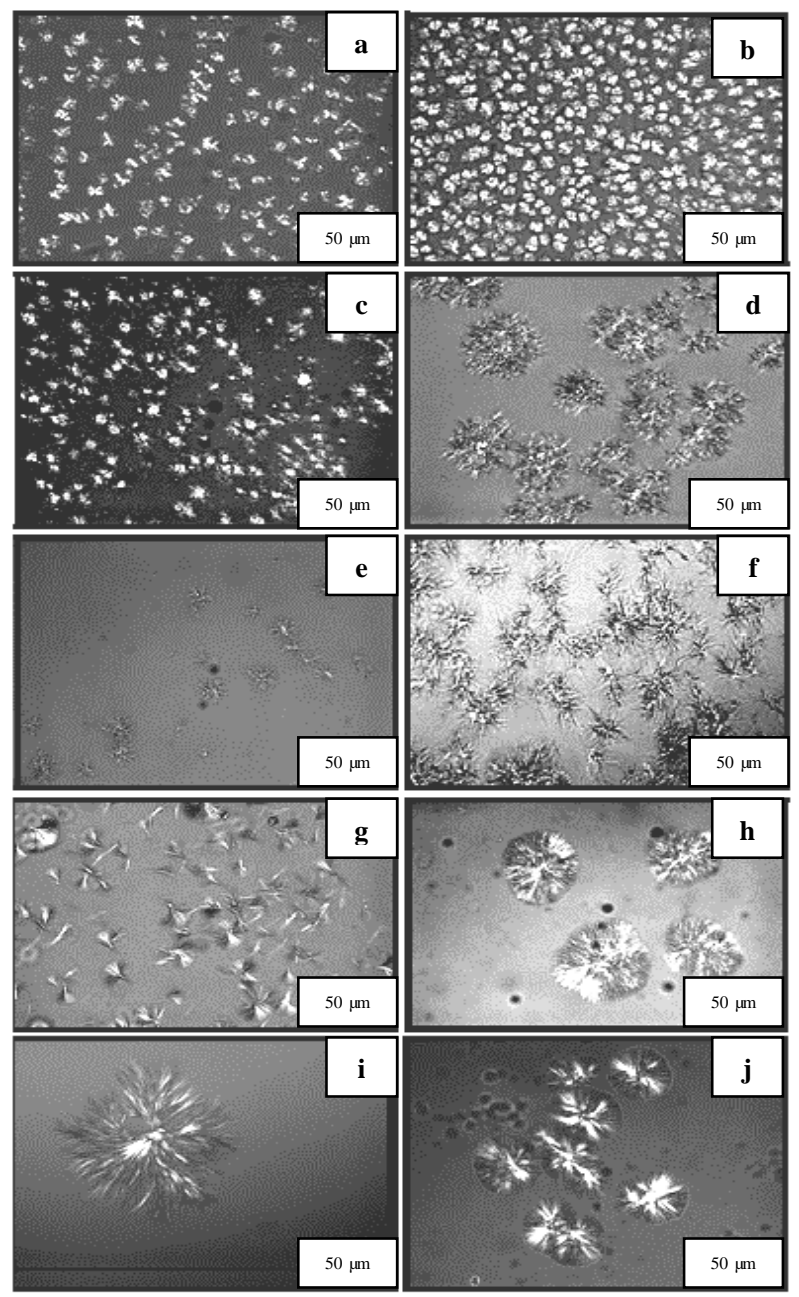

Fig. 3. Microstructural images under PLM at $20^{\circ} \mathrm{C}$ and tempered for $30 \mathrm{~min}$ for PO, PKO and binary blends before and after IE with 200x magnification and magnification bar represent $50 \mu \mathrm{m}$.

Notes: a, NIE PO; b, CIE PO; c, NIE 75PO:25PKO; d, CIE 75PO:25PKO; e, NIE 50PO:50PKO; f, CIE 50PO:50PKO; g, NIE 25PO:75PKO; h, CIE 25PO:75PKO; i, NIE PKO; j, CIE PKO.

\section{CONCLUSION}

Chemical interesterification of PO and binary blends of PO and $\mathrm{PKO}$ produced oil with higher slip melting point and solid fat content due to the randomization of fatty acids in the triacylglycerols. Blending of PO with PKO followed by chemical interesterification altered the crystal types, density and size. This study shows that blending and chemical interesterification are effective modifying the physicochemical properties of the oils. Further study should be carried out on determination of the polymorphic form, crystallization, textural and rheological properties of the blends.

\section{ACKNOWLEDGMENTS}

The authors would like to acknowledge financial support from the FRGS Grant MOHE, Malaysia and technical support from the Malaysian Palm Oil Board (MPOB) and Universiti Teknologi MARA (UiTM), Malaysia.

\section{REFERENCES}

[1] O. Zaliha, C. L. Chong, C. S. Cheow, A. R. Norizzah, and M. J Kellens, "Crystallization properties of palm oil by dry fractionation," Food Chemistry, vol. 86, no. 2, pp. 245-250, 2004.

[2] S. H. M. Fauzi, N. A. Rashid, and O. Zaliha, "Effects of chemical interesterification on the physicochemical, microstructural and thermal properties of palm stearin, palm kernel oil and soybean oil blends," Food Chemistry, vol. 137, pp. 8-17, 2013.

[3] C. W. Chen, C. L. Chong, H. M. Ghazali, and O. M. Lai, "Interpretation of triacylglycerol profiles of palm oil, palm kernel oil and their binary blends," Food Chemistry, vol 100, no. 1, pp. 178-191, 2007.

[4] D. Anderson, "A Primer on oils processing technology," in Bailey's Industrial Oil and Fat Products, Y. H. Hui, Ed. New York: Wiley Interscience, vol. 4, pp. 1-60, 1996.

[5] B. F. Hauman, Tools: Hydrogenation, Interesterification, INFORM, vol. 5, pp. 668-670, 1994.

[6] A. R. Norizzah, C. L. Chong, C. S. Cheow, and O. Zaliha, "Effects of chemical interesterification on physicochemical properties of palm stearin and palm kernel olein blends," Food Chemistry, vol 86, no. 2 pp. 229-235, 2004.

[7] Malaysian Palm Oil Board Test Methods (MPOB), “A compendium of tests on palm oil products, palm kernel products, fatty acids, food-related products and others," Bandar Baru Bangi: Palm Oil Research Institute of Malaysia, 2004.

[8] Z. Zainal and M. S. A. Yusoff, "Enzymatic interesterification of palm stearin and palm kernel olein," Journal of the American Oil Chemists" Society, vol. 76, pp. 1003-1008, 1999.

[9] L. L. Kok, W. R. Fehr, E. G. Hammond, and P. J. White, "Trans free margarine from highly saturated soybean oil," Journal of American Oil Chemists' Society, vol. 76, pp. 1175-1181, 1999.

[10] G. R. List, E. A. Emken, W. F. Kwolek, and T. D. Simpson, "Zero trans" margarines, preparation, structure, and properties of interesterified soybean oil-soy trisaturate blends," Journal of American Oil Chemists' Society, vol. 54, pp. 408-413, 1977.

[11] A. P. B. Ribeiro, R. C. Basso, R. Grimaldi, L. A. Gioielli, and L. A. G. Gonçalves, "Instrumental methods for the evaluation of interesterified fats," Food Analytical Methods, vol. 2, p. 4, pp. 282-302, 2007.

[12] R. E. Timms, "Physical properties of oils and mixtures of oils," Journal of American Oil Chemists' Society, vol. 62, pp. 241-248, 1985.

[13] D. Rousseau, A. R. Hill, and A. R. Marangoni, "Restructuring of butterfat through blending and chemical interesterification. II. Microstructure and polymorphism," Journal of American Oil Chemists' Society, vol. 73, pp. 983-989, 1996.

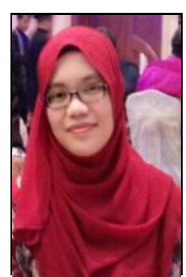

Siti Hazirah Mohamad Fauzi received her M.Sc degree in food technology from Universiti Teknologi MARA, Malaysia and currently working as a research officer in Malaysian Palm Oil Board (MPOB) Malaysia. Her areas of research are interesterification and crystallisation behavior of oils and fats as well as formulating lipid-based coatings for fresh produces. 